Int. J. Dev. Biol. 50: 81-92 (2006)

doi: $10.1387 / \mathrm{ijdb} .052056 \mathrm{lb}$

\title{
Morphomechanics: goals, basic experiments and models
}

"... It is illogical to suppose that in the case of vital organisms, a distinct force exists to produce results perfectly within the reach of physical agencies..."

$$
\text { G. Rainey, cited in D'Arcy Thompson (2000, p. 7) }
$$

\section{Introduction}

A primary role in embryonic morphogenesis of multicellular animals is played by directed and accurately coordinated movements of large cell collectives and by changes in cell shapes. In recent years great progress has been achieved in revealing the molecular mechanics and genetic regulation of these events. Cell movements and shape changes are mediated by contraction of actomyosin filaments associated with membrane dynamics, polymerization of microtubules (Alberts et al., 2004) and periodic changes of osmotic pressure within cells, cell vacuoles and intercellular cavities (Stern, 1984; Beloussov et al., 1993). These events, in turn, are regulated by complicated signaling pathways involving extracellular matrix, integrins, cell junctions, probably mechanosensitive ion channels and Rho machinery (Banes et al., 1995; Chrzanowska-Wodnicka \& Burridge, 1996; Hunter, 2000; Balaban et al., 2001). At least one of the most important cooperative cell movements, namely the so-called convergent cell inter-

\author{
LEV V. BELOUSSOV* and VASSILY I. GRABOVSKY \\ Laboratory of Developmental Biophysics, Department of Embryol- \\ ogy, Faculty of Biology, Moscow State University, Russia
}

calation, requires site-specific expression of the genes involved in a universal Wnt-signaling pathway (Choi, Han, 2002; Wallingford et al., 2002).

These achievements in deciphering the elementary mechanisms of morphogenesis not only do not diminish, but instead renew and increase our interest in the classical problems of a more holistic nature. We ask ourselves why the morphogenetic movements, whatever their molecular mechanisms, are going on during normal development in such a regular and specific spatialtemporal succession? And why, at the same time, does embryonic development exhibit so called structural stability, that is a capacity to reach the normal end-result after substantial rearrangements, removal or addition of embryo parts (embryonic regulation), or even after tremendous variability of its intermediate stages (Kraus, 2006)? Would it be possible to formulate some more or less universal "rules of morphogenesis" permitting us to derive the next step of development from the preceding one being, at the same time, compatible with structural stability?

In searching for ways for solving this task, we have to make a choice between the following two approaches:

Abbreviations used in this paper: CE, contraction-extension feedback; EE extension-extension feedback; GP, growth pulsation; GT, global stretching/ compression threshold; HR, hyper-restoration; MS, mechanical stresses.

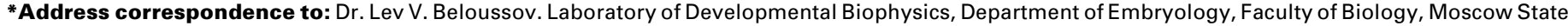
University, Moscow, Russia. Fax: +7-495-939-4309. e-mail: Ibelous@soil.msu.ru ; morphogenesis@yandex.ru
} 
1. A causal chain which provides a transition of a given stage morphological structure to the next stage one, is located exclusively at the molecular-supramolecular level. Briefly speaking, it is a chain of changes in gene expression, linked via signaling pathways, each of which triggers the next one, etc. Within such a framework, the morphological structures (macroscopic shapes) are regarded as mere blind by-products of genetic activity rather than as active members of the causal chains.

2. On the contrary, the shapes of cells and multicellular rudiments are themselves the inevitable and probably decisive links of the developmental causal chains which to a large extent affect the molecular background and hence the next emerging morphological structures.

Paying tribute to refined schemes of genetic interactions (which, in Kauffman's (1993) interpretation, even acquire some holistic properties), one can nevertheless doubt whether they alone are enough for adequately interpreting the main developmental phenomena. Among the main difficulties along this way is the problem of transformation of non-spatial genetic factors into 3-dimensional patterns ["Space (the R3 in our Euclidean geometry) is largely absent from modern biology" (Thom, 1972)]. As shown by recent investigations on Drosophila embryos (Houchmanzadeh et al., 2002; Jaeger et al., 2004), the pattern of gene expression for the next stage can appear in its normal fashion even if a preceding stage was extensively disturbed. This invites us to search for the factors regulating these patterns at a macromorphological level (left intact in these experiments) rather than continuing to believe in the existence of strict causal relations between the patterns themselves. Our next argument is a well known lack of one-to-one correspondence between homologous genes and homologous morphological structures (Wray and Abouleif, 1998): in the different taxonomic groups homologous groups of genes affect the development of largely non-homologous structures and vice versa. Similarly, within a single developing organism, the same sets of regulatory genes can be expressed at different times and in different locations, being associated with quite different types of cell differentiation and morphological structures. This fundamental feature is often defined as a context-dependence of gene action. Although the notion of a "context" is rather vague and includes heterogeneous components, the form of the individual cells and multicellular structures is one of its inevitable components. This comes from numerous evidence on the direct effects of cell shape on the expression of specific genes (e.g., Huang, Ingber, 2000). It is noteworthy that the notion of cell shape includes not only its geometry, but also the topology of cell-cell contacts, whose role in cell differentiation is often decisive (e.g., Arnolds et al., 1983).

But how can shape (or morphology, treated in its broader sense) play such a role? What might be a physical agent, permitting it to affect the underlying molecular machinery which can, in turn, provide the shape for the next stage and so on? Let us remember Thompson's (2000) claim, that "the form of an object is a "diagram of forces"... from it we can judge of or deduce the forces that are acting or have acted upon it". By changing the "forces" to the "stresses" (equilibrated forces divided by the area of their application: in the absence of accelerations such a formulation will be more correct) we fully support this statement and suggest, that there are the mechanical stresses of tension and pressure, closely associated with a given geometric shape, which might be a crucial link in embryonic morphogenesis. This is a central statement of a morphomechanics, a branch of a developmental biology founded long ago by Wilhelm His, D'Arcy Thompson and other authors and encountering now, after decades of neglect, renewed interest (Bereiter-Hahn, 1987; Koel, 1990; Cowin, 2000). Its main tasks consist in studying the generation of mechanical stresses and their role in morphogenesis and cell differentiation (including gene expression). The aim of this paper is to review some general aspects of morphomechanics and to submit some empirical and model evidence demonstrating its heuristic power. For starting this discussion, let us explore briefly the following mechanical model.

Consider an inflated baloon (Fig. 1) with transversal axis $a$ and height $b$. It is proved (Martynov, 1982) that if and only if its transversal axis exceeds its height by no less than a factor of $X X 2$, a series of the vertical folds will appear along the entire perimeter of a body as a result of its inflation, the folds' number being a function of the ratio between a long semi-axis and the shell's thickness. If the body is deflated, the vertical folds will be exchanged with horizontal ones. This example brings us to the following biologically important conclusions:

1. The shape of a stressed body can become complicated (its initial symmetry order diminished) by the action of a homogeneous (in no way prepatterned) force (in our case, that of inflation - deflation).

2. The resulting pattern crucially (although with some redundancy) depends upon the geometry of the initial stage.

3. In stressed shells the orthogonal patterns of stretching compression (so-called Poisson's deformation) will be automatically established, without requiring any special prepatterns.

4. Shape complication of the stressed bodies can be realized, in principle, very rapidly (with the rate of spreading of an elastic wave). On the other hand, some additional rate-limiting mechanisms can be easily introduced.

5. Removal, addition or rearrangement of any part of a body will not violate the end result of its morphogenesis until these perturbations exceed a certain geometric threshold (in the given case, the threshold corresponds to inequality $a<b 2$ ). Similarly, any small enough local deformations of a body will be smoothed out. In the other words, our very much simplified model of a mechanically stressed body shows structural stability.

To summarize: mechanics provides us with a number of universal, definite and essentially holistic rules permitting the generation of more complicated shapes from less complicated ones. On the other hand, if we are trying to generate shapes without the use of mechanics (for example, with the aid of reaction-diffusion models) we need to invent, firstly, some special algorithms for transforming a chemical pattern to a geometrical one and, secondly, to introduce ad hoc for each subsequent structure some new "rules of game" or, at least, some new parameters and/or initial conditions. It would be very strange, if living nature would not use mechanical means for making shapes.

It is but natural therefore that the above described mechanical model has been used for imitating the real biological morphogenesis of the Acetabularia whorl (Martynov, 1982). A similar family of models (Green et al., 1996) reproduced in great details the development of floral rudiments. It is worth mentioning that the genetic components can easily find their places in this kind of model by specifying the values of mechanical parameters (say, 
elasticity) and/or the temporal regimes of the forces' action. For example, they can provide either constant or periodic action of mechanical stresses which is an effective tool for making specific shapes (see below).

Meanwhile, in spite of such advantages, this type of mechanical model cannot reproduce more than one step of morphogenesis, namely, the relaxation of an imposed stress, assuming that the latter is taken as given. Neither generation of an inflation force at the initial time moment, nor further evolution of the achieved shape can be explained within these models' framework. In the other words, the models lacks any feedback, whose existence seems to be an ultimate necessity for any more or less complicated "coming-into-being" (Prigogine, 1980) process, including embryonic development. Is it possible to overcome this deficiency and to create a mechanically-based model containing a morphogenetic feedback? Or, more specifically: would it be possible to consider mechanical stresses as the feedback links between the existing and coming-to-be shapes? The main aim of the subsequent account is to answer this question. By doing this, we will first review what is known about the existence and morphogenetic role of mechanical stresses in embryonic tissues. Then we will discuss several models which include morphomechanical feedback. We will start from some qualitative considerations and then pass to computer models permitting us to make more specific predictions.

\section{Mechanical stresses in developing embryos}

Let us put ourselves a naive question: why an overwhelming majority of developmental events (gastrulation, neurulation, organ formation) are associated with such extensive collective cell movements and transitorial cell shape changes? Instead of precisely coordinating the latter, wouldn't it be easier for a developing embryo firstly to accumulate a required number of cells and then simply to subdivide this cell mass into organs, each one of them being from the very beginning located on its proper place and consisting of cells immediately reaching their final states? At least, according to the concepts of morphogen gradients (see Tsikolia, 2006) and/or positional information such a mechanism would be most reasonable and error-protected. We know, however, that nature avoids it. Even some seeming exceptions, like the development of the morula stages of Cnidarian embryos, exhibit quite extensive, if local, modes of cell deformations and rearrangements (see Kraus, 2006). In our view, this is in no way occasional nor, more importantly, onerous for development. We suggest that morphogenetic movements and cell deformations are indispensable for creating precisely patterned mechanical stresses within embryonic tissues which, in turn, regulate further cell movements and cell differentiation. Let us give a brief review of stressed states in embryonic tissues.

The developing animal embryos of all the species studied in this respect, as well as the growing buds of lower invertebrates (not to say plant rudiments - Green, op cit.) were found to be mechanically stressed (Beloussov et al., 1975; Jacobson \& Gordon, 1976; 1980; Kiehart et al., 2000; see also Kraus, 2006; Cherdantzev, 2006; Cherdantzeva \& Cherdantzev, 2006). This conclusion was made mostly by tracing the immediate deformations of tissues after localized dissections, as well as by other physical and geometrical methods (review: Beloussov, 1998). In lower invertebrates the main source of stresses is the osmotically driven periodic turgor pressure in cell vacuoles (Beloussov et al., 1993), while at the blastula stage of Echinodermata and vertebrate embryos there is a constant pressure within a blastocoel. In amphibian embryos this pressure is about $325 \mathrm{mOsm}=70 \mathrm{~N} / \mathrm{cm}^{2}$ (Wilson et al., 1989). The tangential tensile stress $S$ of a hemispherical shell (blastocoel roof) may be calculated by a standard formula, $S=r P /(2 t)$, where $r$ is the radius of a hemosphere, $P$ is the pressure and $t$ is the thickness of the roof. By optical microscopy data $t$ XX $100 \mu \mathrm{m}$ and thus $S \times X 200 \mathrm{~N} / \mathrm{cm}^{2}$, which is a relatively small value. However, if we take into consideration that the turgor-produced tension is resisted by nothing more than few focal junctions with a total cross section of no more than $t \mathrm{XX}$ $10 \mu \mathrm{m}$, the effective thickness should be about 10-fold reduced and hence tensile stress 10 -fold increased. Taking a cross section of an actin filament as $2.5 \times 10-13 \mathrm{~cm}^{2}$, we obtain about $10-10 \mathrm{~N}$ per filament (which exceeds the estimate by Balaban et al., 2001, on the single cells by no more than an order of magnitude). The effects of such forces on the molecular level cannot be neglected. At the advanced stages of development the stresses are produced mostly by cooperative cell movements of involution (during gastrulation) or neural tube rolling. They have values of the same order (Beloussov, 1998). Most important is that they are effectively transmitted via actin and intermediate filaments, cell-cell contacts and extracellular matrix along distances comparable with the overall body dimensions. As a result, quite regular patterns (fields) of mechanical stresses are established within entire embryonic bodies, remaining topologically constant during rather prolonged periods of development (for amphibian embryos: from blastula to early gastrula stage; from early to late gastrula stage; and so on) (Beloussov et al., 1975). The main elements of the stress fields are the lines (surfaces) of the maximal tensions, tension nodules (meeting points of differently oriented tension lines) and the pressurized compartments surrounded by tensed surfaces. The presence of nodules indicates the existence of tension gradients (gradients of a "tension density") with the highest points coinciding with nodules.

The existence of tensile stresses is a necessary condition for the organized movements of cells and hence for morphogenesis. Here are three examples related to quite different objects:

1. Presence of an elastic substrate, flexible enough to be

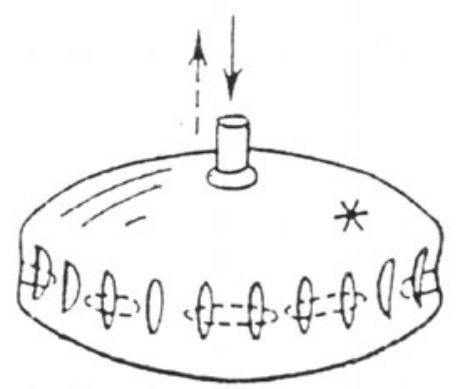

Fig. 1. Inflation (solid arrow) of a flattened balloon generates on its surface a series of vertical folds, while its deflation (dotted arrow) produces a series of horizontal folds. In each case, the number of folds is quite precise and depends upon the balloon's geometry. This shows how a regular complicated pattern can be created by applying no more than one volume changing force. From Martynov, 1982, with the author's permission. 

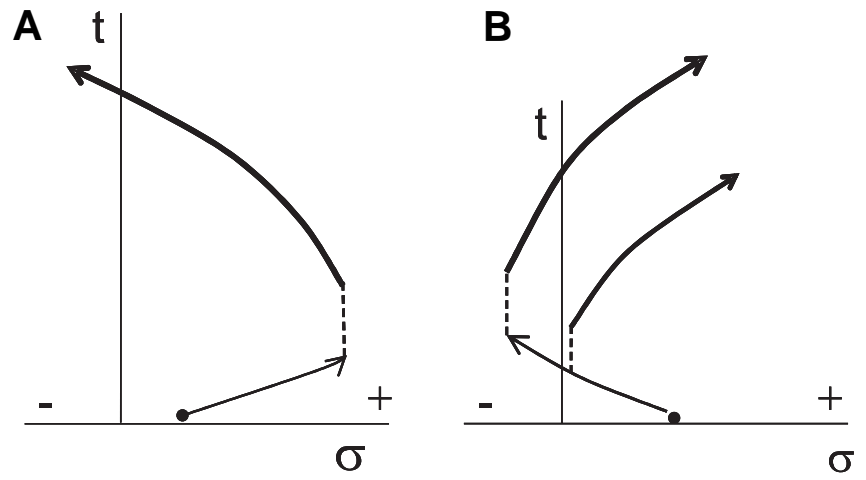

Fig. 2. Schemes of hyper-restoration (HR) responses to the shifts of mechanical stresses from the initial values denoted by black spots. Fine lines indicate the passive stresses, that is, the actions of external stretching (A) or relaxation/compression (B) forces while solid lines indicate the active responses to these forces. Vertical dashed lines indicate lag periods between stabilization of an external force and the start of an active response to it. Horizontal axes: stress values (positive tensile stresses to the right, negative pressure stresses to the left). Vertical axes: time.

stretched by substrate-attached cells, is a necessary and sufficient condition for providing in vitro emergence of regular cell patterns (Harris et al., 1984).

2. Self-stretching of the chick embryonic disk at the beginning of incubation is necessary for its further development (Kucera and Monnet-Tschudi, 1987).

3. Presence of a turgor pressure either within a gastral cavity (fresh-water hydra buds: Wanek et al., 1980) or within cell vacuoles (marine Hydrozoa: Beloussov et al., 1993) is necessary for growth and shape changes of the buds.

It was shown also, that relaxation (even if temporary) of the internally generated mechanical stresses leads to the grave anomalies in the development of chicken embryos (Bellairs et al., 1967), eye rudiment (Coulombre, 1956) and amphibian embryos relaxed at the blastula early gastrula stages (Beloussov et al., 1993, 1994; Ermakov and Beloussov, 1998). In the latter case it was found that the relaxation of stresses, while not preventing cell differentiation and the formation of the main body rudiments, largely increases the variability of the rudiments' volumes and the

Fig. 3. HR responses in bodies of different geometries. $\left(\mathbf{A}_{\mathbf{1}}\right)$ Passively bent cell layer with its convex surface stretched and the concave one compressed. $\left(\mathbf{A}_{\mathbf{2}}\right)$ Its expected response, namely the active expansion of the convex surface and the active contraction of the concave one. Bent arrows within a layer indicate the expected flow of cell material. (B) A lip shown in saggital section, combining the $\left(A_{2}\right)$ response with the involution of cell material from the extended convex to the contracted concave surface. $\left(\mathbf{C}_{1}\right)$ A pressurized multicellular torus. Expected is a latitudinal cell convergence (converged arrow) relaxing dominating meridional stresses (vertical bidirectional arrow). $\left(\mathbf{C}_{2}\right)$ Details of cell convergence shown onto a flat projection of a toroidal surface. $\left(\mathbf{C}_{\mathbf{3}}\right)$ As a result, a former torus approaches a sphere with a diminished central hole. $\left(\mathbf{C}_{\mathbf{4}}\right)$ In the case of an overshoot of cell convergence, a torus can be transformed into a tubular structure. $\left(\mathbf{C}_{\mathbf{5}}\right)$ A similar structure grown during $20 \mathrm{~h}$ from an isolated toroidal marginal zone extirpated from a X. laevis early gastrula embryo. (D) A 3-dimensional view of a lip, showing cell convergence towards its midline (a "funnel" effect). asymmetry index $\mathrm{A}=\left(\mathrm{V}_{1}-\mathrm{V}_{2}\right) /\left(\mathrm{V}_{1}+\mathrm{V}_{2}\right)$ of the total volumes $\mathrm{V}$ of the opposite somite series. For relaxed embryos $A=0.37 \pm$ 0.06 , to be compared with only $0.05 \pm 0.01$ for normal embryos. Therefore, normal stress patterns are indispensable for providing the proportionality and symmetry of the rudiments.

No less important is the role played by stress patterns in the viability and differentiation of individual cells. Under conditions preventing cells' self-stretching the apoptotic program is switchedon while cell type specific genes are inhibited (Huang and Ingber, 2000).

\section{Stress-mediated developmental feedback and a hy- pothesis of hyper-restoration of mechanical stress values}

Stress-generating molecular machines are themselves mechanosensitive (Banes et al., 1995; Chrzanowska-Wodnicka \& Burridge, 1996). For example, stretching of a tissue sample leads to the assembly of microfilaments (Kolega, 1986) and microtubules (see below) from their subunits and, in the case of Physarum cytoplasmic strands (Wohlfarth-Bottermann, 1987), to the contraction of the just assembled microfilaments. On the other hand, relaxation of tensions promotes the disassembly of cytoskeletal elements. These features create a basis for regarding mechanical stresses as important links in several morphogenetic feedbacks. To the best of our knowledge, the first model exploiting such a possibility was suggested by Odell et al. (1981). The authors postulated that the passive mechanical stresses (in the sense of those originating outside a given part of an embryo and/ or before a given time period) could be coupled by feedback to active forces (that are generated locally at the given time period). Their specific proposal was that passive tangential stretching of part of an epithelial layer can trigger active contraction of this part, which will in turn stretch the next part of this cell layer, thereby
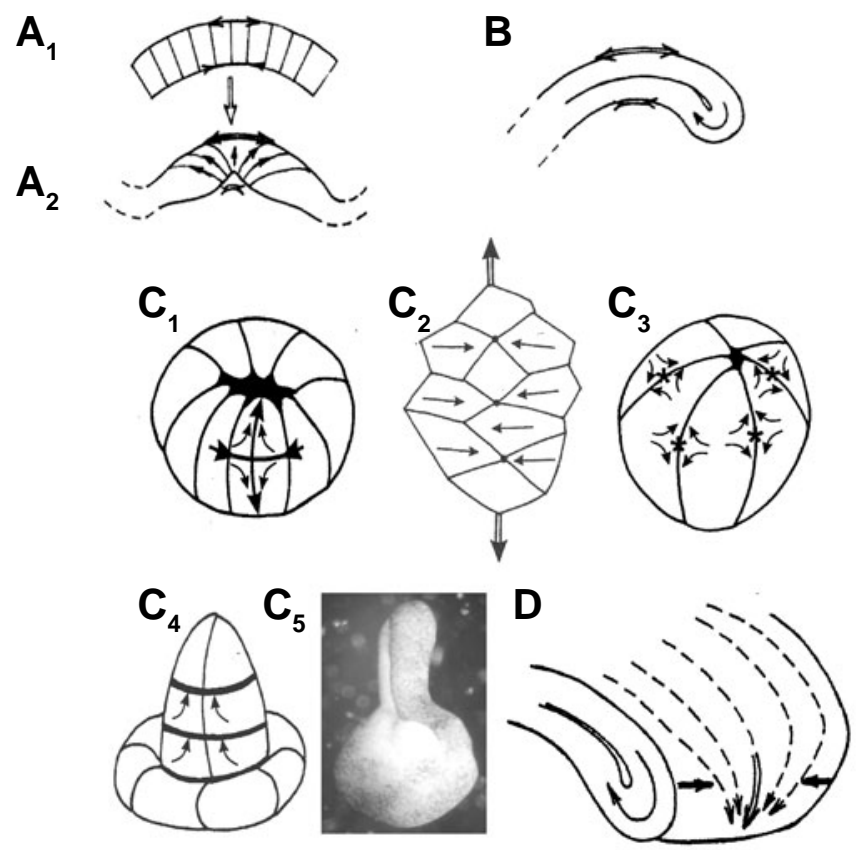
producing long-range "+, +" feedback coordinating and synchronizing such processes as gastrulation and neurulation. Let us call this reaction the "Odell's response". On the other hand, Belintzev et al. (1987) made a suggestion which looks at the first glance to be the opposite. Namely, they presented evidence that the relaxation of tangential tensions, rather than increase of these tensions, could initiate a cooperative process of cell columnarization thus providing tangential contraction of a given cell layer.

Today we propose that the both reactions can take place, as depending upon the given mechanical conditions and that they can be embraced, together with several others, by a more general rule which we define as a hypothesis of stress hyper-restoration (HR hypothesis) (Beloussov et al., 1994), as follows:

Whenever a change is produced in the amount of local stress applied to a cell or local region of tissue (regardless of whether this change in force comes from a neighboring part of the embryo or has been exerted by an experimenter), the cells or tissue will respond by actively generating forces directed toward the restoration of the initial stress value, but as a rule overshooting it. Whenever such changes in stress are unevenly distributed (stronger one place than another), or are anisotropic (stronger in one direction) then the responses induced will be directed towards reducing (with an over-shoot) whichever deviations were greatest.

For example, if a sample is stretched by an external force, it tends to diminish actively the stretching even up the point of generating the internal pressure stresses (Fig. 2A). On the other hand, if a sample is relaxed or even compressed, it tends to increase its tangential tension up to and exceeding the initial value (Fig. 2B). There is also some lag period between the action of an external force and the active samples' response to it. The durations of the lag periods and the rates of the active responses may be regulated both genetically and epigenetically.

Let us formulate some expectations and predictions coming from this hypothesis and compare it with experimental results.

First of all, it is easy to show, that the ways for reaching the definite end-results will depend upon the firmness of fixation of the sample's edges after its deformation by external force. Namely, if after the end of stretching at least one of the edges is not firmly fixed, the Odell's response, that is, the sample's contraction, is possible. However, usually in developing embryos the reverse is true fixation of the stretched part's edges is too rigid to be overridden by any internal contractile forces. Under these conditions quite another reaction can be used for effectively transforming an external stretching to an internal pressure stress. This is the contraction of a sample's material in the direction perpendicular to a stretch and hence (due to the material's incompressibility) the active samples' extension in the stretch direction. In a multicellular sample this reaction can be realized either by the active deformations of individual cells or by cell-cell insertion (intercalation) in the direction perpendicular to a stretch.
Correspondingly, if the relaxation is not followed by a firm fixation of the relaxed sample's edges, the latter can restore and even hyper-restore its initial tension value by spreading (extending) along a substrate. Again, however, this condition is rarely realized in embryonic tissues. Meanwhile, if the relaxation is followed by the firm edges' fixation (which is more usual), the only way for (hyper) restoring the tensions would be a sample's contraction in the direction of a previous stretch. This can be achieved either by a direct tangential contraction (columnarization) of cells in accordance with the Belintzev et al. (1987) model and/ or by migration of some cells inside the sample.

Very often both in the individual cells and embryonic tissues the tensile stresses are coming from a focused source and then diverged throughout more extended tissue areas, creating what may be called tension gradients. In these cases $\mathrm{HR}$ reactions are expected to go in a way not only smoothing out, but even reversing the latter. This can be done by extending the area of a focused source. In the individual cells this can be made by enlargement of stress-bearing cell-cell contact areas, while in multicellular tissues the same result can be achieved by cell migration towards the focus area (that is, uphill the tension gradient). This latter event we suggest calling a "tensotaxis" (Beloussov et al., 2000).

Now let us look what the HR hypothesis may predict for samples with a bit more complicated geometry. Consider first a part of a cell layer bent by external forces. Correspondingly, its convex side will be stretched and the concave side compressed (Fig. $3 A_{1}$ ). Similarly to the case of stretching, we have here two possibilities depending upon the rigidity of the edge fixation. If the edges are relieved, a bending layer should behave as a "reinforced string", not only flattening, but even curving itself to another side. If, however, (as usually takes place) the edges are firmly fixed, the expected $\mathrm{HR}$ reaction would be the active extension of the convex side and the active contraction of the concave one, both of which could be simultaneously accomplished by migration of cells from the concave to the convex side. This will magnify what begun initially as passive bending (Fig. $\left.3 \mathrm{~A}_{2}\right)$.

Next, consider the prevalent category of embryonic structures, called a "lip", in the sense of the lips of a blastopore. As viewed in saggital section (Fig. 3B), this is a slightly curved fold. As was just
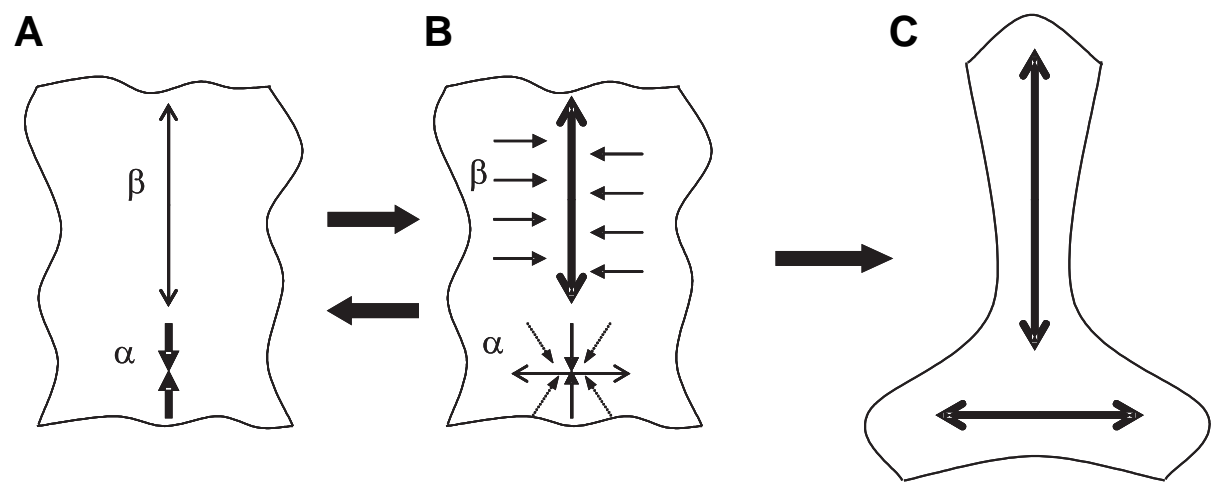

Fig. 4. A scheme of contraction-extension (CE) feedback. (A) A sample (cell layer) with its part $\alpha$ actively contracted and hence part $\beta$ passively stretched. (B) Part $\beta$ responds to stretching by active (most probably, intercalation-mediated) extension which relaxes/compresses part $\alpha$ in the vertical direction and extends it horizontally. States $A$ and $B$ can alternate several times. (C) $A$ passive horizontal extension of the $\alpha$ region can stimulate cell intercalation ( $B$, dotted arrows), so that two mutually orthogonal zones of cell intercalation arise. 
discussed above, the concave side of such a lip should contract actively, while the convex one should expand. Furthermore, if these changes in tension are accomplished by migrations of cells from one surface to another around the lip circumference, the result will be an involution (Fig. 3B, bent arrow).

As a third example, we consider a torus, or any part of a toroidal surface. As is known from mechanics (Landau \& Livshitz, 1976), the meridional tensions of a stressed torus (pressurized from inside) are greater than the latitudinal ones. Therefore, in order to hyper-relax the greater tensions, the flow of material (the migration of cells) should be directed towards the meridians (Fig. $3 \mathrm{C}_{1}$ $\mathrm{C}_{3}$ ). The result for a torus will be first to approach towards the shape of a sphere, with the narrowing of its central hole and then (in the case of an extensive overshoot) to a tubular body extended perpendicularly to what had been the plane of the torus (Fig. $\left.3 \mathrm{C}_{4}\right)$. An example is given on Fig. $3 C_{5}$.

Now let us note that in a 3-dimensional view a "lip" is a part of a semi-toroidal surface. This will cause such a lip to behave as a sort of "funnel", simply because of its geometry, which simultaneously provides for its involution and for the convergence of cells towards the location of this involution (Fig. 3D).

Another important property of HR reactions is that they can automatically become coupled with one another by means of positive feedback cycles. The two most important cycles can be called contraction-extension (CE) feedback and extension-extension (EE) feedback.

CE feedback can be initiated either by the active contraction of part of a tissue ( $\alpha$, Fig. 4 A,B) or by the active extension of another part $(\beta)$. The result of contraction at the $\alpha$ location will be passive stretching of the adjacent $(\beta)$ tissues, which responds by an active extension, probably mediated by cell intercalation. This extension will at least relax the tension in part $\alpha$ and, if strong enough,
A
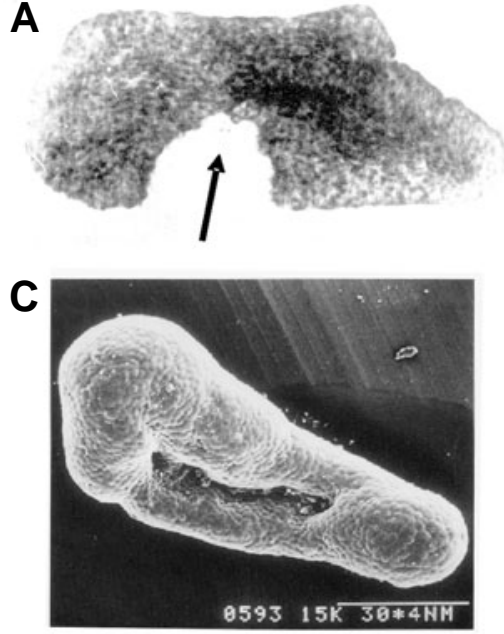

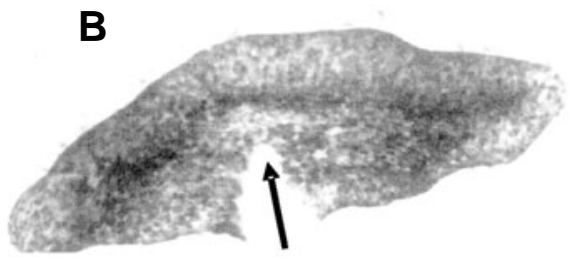

D

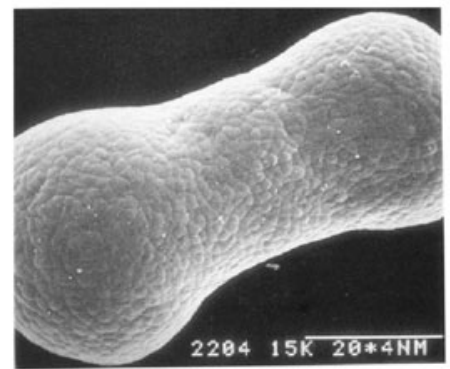

Fig. 5. Results of stretching the explants of ventral ectoderm extirpated from early gastrula $\boldsymbol{X}$. laevis embryos onto an adhesive substrate. (A,B) In vivo explants, 2 min after making incisions (arrows). (A) After cessation of $30 \mathrm{~min}$ stretch. Wide gap indicates substantial tension. (B) A similar explant $2 \mathrm{~h}$ later with no additional stretch. Note autonomous elongation as compared with (A), convergence of pigmented cells towards the explant's longitudinal axis and diminished tensions indicated by a reduced gap. (C,D) Explants 5-6 h after the end of a stretch. Formation of polar inflations points to the generation of internal pressure (from Beloussov et al., 2000). produce compression there. That will, in turn, promote the next round of active contraction in the $\alpha$ location, which will again stretch part $\beta$ and so on. In the case of a substantial compression of the $\alpha$ part, it will be first passively and then actively, extended in the perpendicular direction, creating together with the $\beta$ part an orthogonal pattern (Fig. 4C). It is easy to see that just the same sequence of events can be initiated by the active extension of the $\beta$ part, rather than contraction of the $\alpha$ part.

CE feedback can also act along the circumference of a torus, if the latter's contraction is resisted by any external force. Under these conditions one or few meridians (as depending upon the the main axes of cell convergence.

EE feedback comes into action when two or more cells or cell layers contact each other's lateral surfaces that are able to extend, both passively and actively. Active extension of one of these will cause stretching of the neighboring one, which will respond by an active extension along the same geometric axis as the force that stimulated it. A succession of this kind of passiveactive response can propagate in a relay fashion through an entire collection of samples and return back to the first ones, keeping them in an extended state.

\section{Empirical evidence of $\mathrm{HR}$ reactions}

\section{Multicellular level}

Stretch-promoted cell intercalation. If a piece of the suprablastoporal zone of an early gastrula Xenopus embryo is tched onto an adhesive substrate perpendicularly to its noringly reorient the direction of their intercalation and as a result the piece will actively extend in the direction of its previous stretching (Beloussov et al., 1994). The same reaction takes place in a piece of ventral ectoderm stretched in an arbitrary direction (Fig. 5A-D). Within several hours after the end of a stretch the pieces take a dumb-bell shape with inflations at both poles, indicating that they are under internal pressure (Fig. 5 C,D). Transfer of an external tension to the internal pressure clearly indicates an overshoot reaction similar to that shown in Fig. $2 \mathrm{~A}$.

Relaxation-promoted cell columnarization. A usual reaction to the relaxation of the normal tangential tensions of embryonic tissue is its tangential contraction, producing one or several coherent domains of highly columnarized (sometimes bottle-shaped) cells (Beloussov et al., 1994). For example, such a domain can be produced in the ventral ectoderm of the early gastrula Xenopus laevis embryo as a result of the relaxation of circumferential tensions (Beloussov etal., 2006). The overshoot increase of tangential tensions after this relaxation can be traced by evaluating the bending angles of separated tissue pieces before the operation (Fig. 6A), immediately after the relaxation (Fig. 6B) and 40 min later (Fig. 6C). One can easily 
A
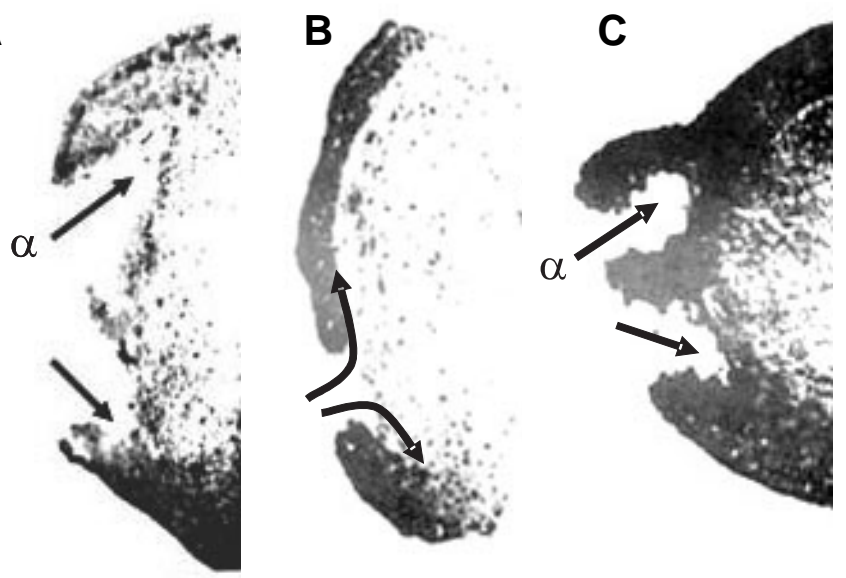

Fig. 6. A comparison of the bending angles $(\alpha)$ formed by the dissected edges (arrows) of an ectodermal layer in the intact $\mathrm{X}$. laevis early gastrula (A), a few minutes after relaxation of the tangential tensions by a ventral incision of an ectodermal sheet (B) and about $1 \mathrm{~h}$ later (C). In all cases, embryos were fixed 1 min after dissection. The bending angles in (C) exceed those observed not only in (B), but also in (C), pointing thus to a hyperrestoration of the tensions taking place within an hour after their relaxation. Sections are deliberately thick $(20 \mu \mathrm{m})$. From Beloussov (1998).

see that the latter angles exceed not only the second, but also the first ones. Insofar as the value of the bending angles is roughly proportional to the tension on the apical cell membranes (Beloussov et al., 1975), the tension at 40 min after relaxation overshoots the pre-relaxation tension. For other overshoot examples see Beloussov et al., 2006.

\section{Individual cell level}

Some elegant examples of overshoot reactions to mechanical stresses can be gleaned from single cells studies. Such are, for example, the stretch and relaxation responses in growing axons (Buxbaum \& Heidemann, 1992). Namely, within a certain stretching range neurites respond to tension as passive viscoelastic bodies, whereas at a stretching threshold of 100 microdynes, an active elongation response, sometimes associated with a marked lowering of tension, was observed; on the other hand, after sudden diminution of tension, neurites contract, sometimes doubling the pre-experimental steady-state tension. These responses are probably associated with the polymerization-depolymerization of microtubules. A stimulation of microtubule growth by tension has been demonstrated elsewhere (Kaverina et al., 2002). Kinetochore microtubules of a mitotic spindle are also under tension (Skibbens et al., 1994), caused by kinesin- and dynein-mediated dynamics of the polar and astral microtubules. The tension is necessary for inserting further tubulin subunits into the kinetochore microtubules (Alberts et al., 2004). This may be regarded as a close molecular analogue of a tension-promoted cell intercalation.

Another set of stress-dependent cell reactions is associated with membrane dynamics. Several examples indicate that the relaxation of the cell membrane stimulates endocytosis (Ivanenkov et al., 1990) while its stretching promotes exocytosis (Wirtz \& Dobbs, 1990) and growth of the intercellular contact zones (Pletjushkina et al., 1998; Riveline et al., 2001). Obviously, the relaxation-promoted endocytosis is directed towards tension in- crease, while stretch-promoted exocytosis and increase of cellcell contact areas towards the decrease of tensions (since the tension values are inversely proportional to the contact areas). Therefore, the direction of the responses is compatible with our hypothesis.

In a detailed micromechanic study (Balaban et al., 2001) a roughly linear proportionality has been described between the contractile force applied to a single focal junction and the junction's area. This proportionality is established quite rapidly, no more than in few seconds after the change of contractile force value. The maintenance of proportionality means that the constant value of the tensile stress value is accurately kept, what corresponds to the limiting case of our central suggestion (a feedback without an overshoot). It would be of interest to know, whether in this experimental system the overshoot can nevertheless be achieved after a more prolonged force application.

\section{Models of morphogenesis based upon the HR hypoth- esis}

We formulated the HR hypothesis in an deliberately loose way, leaving much freedom for its specification by ascertaining the values of the implicated parameters. To these belong, for example, the critical values of the externally applied stresses required for switching-on the active sample's responses, the rates and values of the responses themselves and, as a special category, the temporal patterns of either passive or active stresses. For example, the stresses can be applied either constantly, or with some periodicity, etc. It is biologically plausible to suggest that these parameters values are regulated both epigenetically and genetically, providing the production of diverse patterns and shapes. We have used a model approach for exploring the morphogenetic consequences of parametric changes. In this section a brief description of two such interrelated models is presented (for more detailed account see Beloussov and Grabovsky, 2003, 2005).
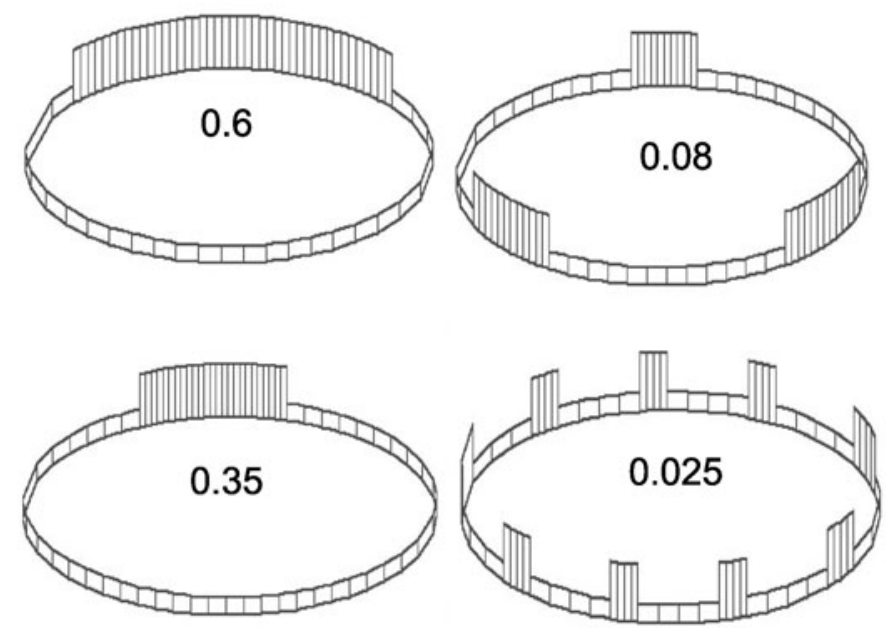

Fig. 7. Generation of one-domain and multi-domain (metameric) patterns via different values of the global threshold (GT) parameter (shown by figures). Diminishing the GT value decreases a single domain length and increases the total number of domains. 
A

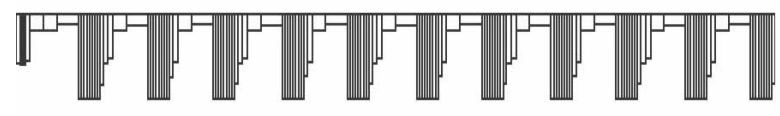

B

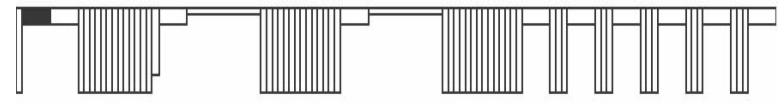

C

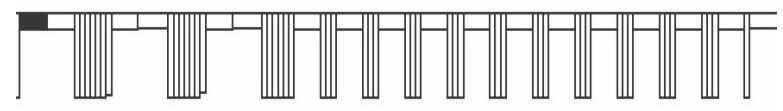

D

$\mathbf{E}$
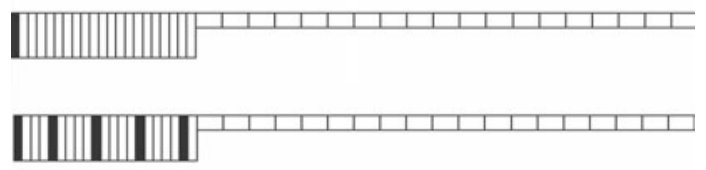

$\mathbf{F}$

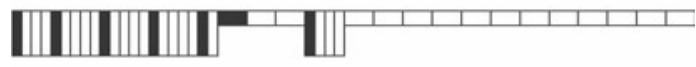

Fig. 8. Patterns emerged on a row of homogeneous cells (filled are those initially excited to become columnar). (A) Homonomic segmentation. (B,C) Two examples of heteronomic segmentation. In (B), cell number is twice as great as in $(A)$, all the parameters are the same. In (C) the parameter controlling the rate of cell columnarization is increased. (DF) illustrate a structural stability of domain formation under different numbers and location of initially excited cells. In all cases, the largest columnar domain consists of 21 cell. For more details see Beloussov and Grabovsky, in press.

\section{Models of CE and EE feedback in a cell layer of zero or uniform curvature}

We consider a uni-dimensional row of isodiametric cells closed into a circle of a constant length (cell number is also taken constant throughout the entire modeling). It may imitate either a torus, or a cell sheet of a zero or uniform curvature. The initial configuration is taken to be unstressed. The modeling starts by applying to cell \# 1 a certain value of tangential contraction (with its square remaining constant; hence, this cell becomes more columnar). As a result, the entire layer's surface will be passively stretched. According to the HR hypothesis we assume that a certain (critical) amount of the passive stretching should induce the active extension response, while a critical amount of relaxation/compression should generate the active contraction response. The main task of the model is to explore the morphogenetic consequences of the changes in these critical stress values. For doing this, we introduce a global stretching/compression threshold (GT), which can vary from 0 to 1 but is kept constant within the entire model time. We assume that if and only if a stretching or compression stress applied to a given cell exceeds the threshold, a cell generates (according to CE feedback idea) the active extension or contraction response, respectively. The active responses are assumed to go in a relay fashion (say, in a clock-wise direction) from one cell to another with a rate much slower than the spreading of the passive stresses. An indefinite number of relay successions is allowed. Different values of the active responses can be also introduced as independent parameters.

In addition, we suggest that besides a global stress, each cell A "perceives" the shapes of the both its neighboring cells (B and C). Namely, if the arithmetic mean of heights/width proportions of $B$ and $C$ is greater than 1 (that is, $B$ and $C$ are inclined to become columnar) the same will take place with cell $A$ and vice versa. In the other words, any cell is "infected" by its neighbors to take the same shape. That fits the idea of EE feedback. The main results of the modeling were the following.

1. The decisive parameter for the modeled patterns is the GT value. If it is high enough (close to 1), a cell layer becomes segregated into single domains of columnar and flattened cells (Fig. 7A). Under progressive GT diminishment, the length of the columnar domain first becomes reduced and then splits into an increased number of smaller domains (Fig. 7B-D). Therefore, CE feedback can produce, depending upon the parameter's values, quite different patterns, starting from a single extended domain of columnar cells (similar to a neural plate, or to sensory placodes) up to segmented (metameric) patterns. Among the latter, not only a homonomic pattern (all the segments the same) but also a heteronomic one (some number of "anterior" segments differs from the "posterior" ones) can be reproduced by increasing the value of the active cells' extension (Fig. 8 A-C).

2. Patterns produced under fixed GT values are almost independent from the size of (number of cells within) the model row. Therefore, the model fairly well reproduces a universal phenomenon of scaling (embryonic regulation).

3. The modeled patterns are, to a finite extent, structurally stable. For example, same size columnar cell domain will be formed, whether either one or several not too far located cells are initially perturbed to become columnar (Fig. 8, cf D and E). If adding to them two more excited cells, a next small domain is formed, but the first one retains its length even at the expense of one excited cell (\# 6 from the left) to become flattened (Fig. 8F). These results illustrate again the holistic properties of the model and have numerous biological parallels.

4. Under very low GT values and/or under high values of the active extension, response of the arisen patterns become nonstationary and they are transformed into perpetually running waves of cell contraction-extension. Therefore, the stationary structures and propagating waves can be reproduced within the same model framework.

Therefore, the combination of CE and EE feedback has wide generative capacities, able to produce quite diverse patterns, including non-stationary ones. It is also worth mentioning, that the parameters employed can be spread homogeneously throughout the entire system. That facilitates parametric regulation both by genetic and epigenetic mechanisms.

\section{Curvature increasing models}

The aim of this family of models was to reproduce morphogenetic consequences of curvature-increasing feedback (Fig. $3 \mathrm{~A}_{1}-$ $\mathrm{A}_{2}$ ) by taking into consideration several biologically plausible parameters. By doing this, we had in mind, first of all, the morphogenesis of hydroid polyps from the Thecate subdivision, although the main model's ideas are applicable to other taxonomic groups as well. Morphogenesis of the buds of hydroid polyps starts from geometrically simple shapes which can be 
adequately approximated by cusps joined with cylinders (Fig. 9A). Growth and shaping goes on as a series of strictly periodic, osmotically-driven pressure impulses obeying quite regular stageand species-specific temporal patterns (growth pulsations, GP: Beloussov et al.,1993). Due to these impulses the buds stretch the covering visco-elastic layer, the so-called perisarc and acquire the resulting shapes. In our model we reproduced a regime of constant pressure (Fig. 9B $\mathrm{B}_{1}$ ), as well as different biologically realistic GP patterns (Fig. $9 \mathrm{~B}_{2}$ and $\mathrm{B}_{3}$ ). Also, we introduce the elasticity parameter $W$ (a measure of resistance to any deformations) which can vary from 0 to 1 but remains constant within the entire given modeling time. The main conclusions from the model are briefly the following (for more details see Beloussov and Grabovsky, 2003):

1. Within a certain range of the ratio $W / G P$ shapes quite similar to the real ones are imitated (Fig. 9 C,D). Outside this range, the increase of $W$ value leads to nothing more than a smooth unfolding of initial shapes (Fig. 9E) while its decrease leads to the condensation of the model points into a single spot (Fig. 9F).

2. Periodicity of the pressure impulses turned out to be crucial for creating realistic macroscopic shapes with smooth contours (Fig. 9 B2,B3). If, on the contrary, the curvature-increasing forces acting permanently, just a poor set of monotonously indented shapes is reproduced (Fig. 9B1). Therefore, the temporal patterns of the deforming forces are indispensable and powerful tools for making specific shapes even in the case of complete spatial uniformity of the forces' distribution.

We have also explored whether the same model can be used for generating biologically realistic shapes with the definite order of radial symmetry from circular shapes. In these constructions, we took a curvature-increasing force starting to act from a single point of a circle and then rotating with a definite handedness. Again, under constant (non-periodic) force nothing except monotonously indented "rack-wheels" was reproduced (Fig. 10A). Meanwhile, if we introduced any kind of a periodicity, quite realistic and ordered shapes could be obtained, again greatly dependent upon the parameters' values. Besides the $W$ parameter, the number $N$ of (the relative distance between) the kinematically independent elements of the initial circle now be-

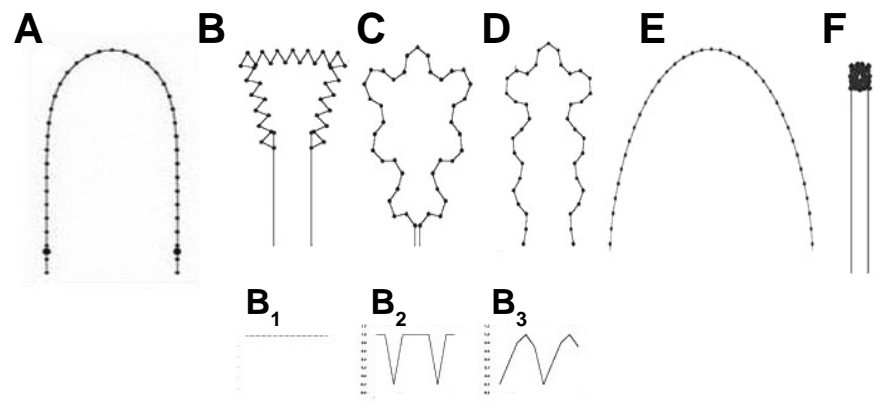

Fig. 9. Shapes produced by curvature increasing model. (B-D) Shapes generated from those shown in (A) under the regime of a constant pressure $\left.\mathbf{B}_{\mathbf{1}}\right)$ and the different regimes of the pressure impulses $\mathbf{B}_{\mathbf{2}}-$ $\left.\mathbf{B}_{3}\right)$. For $(B-D)$, the parameter $W$ takes the values of $0.45,0.48$ and 0.39 correspondingly. (E) Under high $\mathrm{W}$ values $\mathrm{W}=0.7$ ), nothing except a smooth unfolding of the initial contour takes place. (F) Under low W values ( $\mathrm{W}=0.1)$ the model points are clustered into a dense spot (from Beloussov and Grabovsky, 2003).
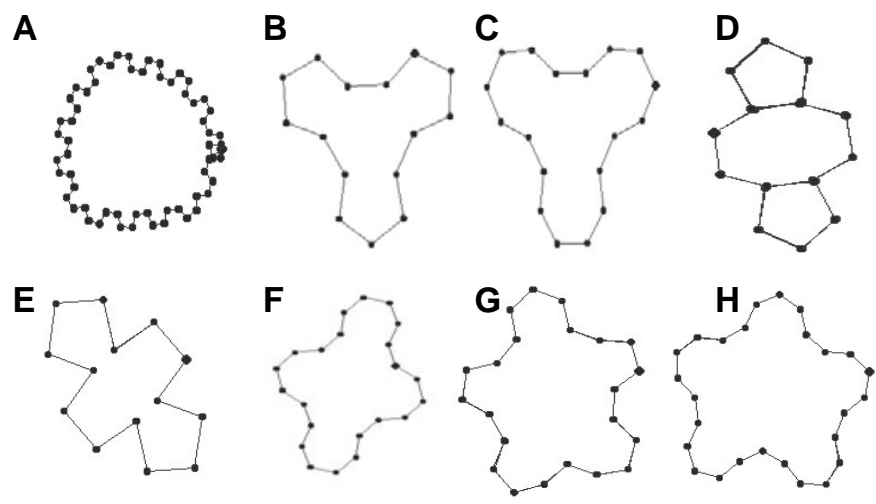

Fig. 10. Formative capacities of circular shapes under different $\boldsymbol{N}$ and $\boldsymbol{W}$ values. (A) A monotonous "rack-wheel" is produced under the action of a constant (non-periodic) force at any $\mathrm{N}$ and $\mathrm{W}$ values. (B-H) Radial shapes containing from 3 to 5 lobes, produced under periodic pressure pattern shown in Fig. $7 B_{1}$. (B) $N=15, W=0.47 ;$ (C) $N=18, W$ $=0.49 ;$ (D) $\mathrm{N}=14, \mathrm{~W}=0.41 ;$ (E) $\mathrm{N}=18, \mathrm{~W}=0.47 ;$ (F) $\mathrm{N}=22, \mathrm{~W}=0.47$; (G) $\mathrm{N}=22, \mathrm{~W}=0.47 ; \mathbf{( H )} \mathrm{N}=26, \mathrm{~W}=0.49$. Note that quite similar shapes can be produced under different combinations of both parameters.

came crucial. The parameter $N$ can be regarded as a reverse measure of half lengths of generic undulation waves of a given body (Green et al.,1996). Although most of the shapes obtained under different parameters values turned out to be asymmetric and unstable (perpetually rotating), some remarkable "islands of stability" could be traced, where within the finite range of $W$ and $N$ parameters the regular radial patterns with definite numbers of lobes emerged (Fig. $10 \mathrm{~B}-\mathrm{H}$ ). These results highlight possible relations between the invariable morphomechanical laws and the parameters' values, which could be set genetically or epigenetically. Being necessary for determining definite shapes, these latter do not contain perse (out the context of morphomechanical laws) any "developmental information". If we know indeed only the parameters' values and do not know the morphomechanical rules, we know virtually nothing about shapes; on the other hand, if we know only the morphomechanical rules, at least a set of potentially possible shapes can be reproduced, even if we cannot predict which one of them will be realized. Let us also emphasize the redundancy of the "parametric information": very similar shapes (for example: Fig 10 B,C; D,E,F; G,H) can be reproduced under different combinations of the parameters $W$ and $N$.

\section{Discussion}

The morphomechanical approach outlined in this paper is in tune with modern views on the nature of the signaling pathways and gene expression. What we mean is the assumption that the "pathways... may signal in a semi-solid state fashion with minimal free diffusion" (Hunter, 2000). Accordingly, the mechanical stresses should be regarded as inevitable links in the signaling pathways. Interestingly, even intracellular water is regarded to be mostly in a bound state endowed with such mechanical properties as "osmotic tension" (Watterson, 2004). The intercellular space is also highly structured, with several layers of water bound to the extracellular matrix components. As a result, continuous mechanically deformable pathways are going from extracellular matrix via integrins, clustered membrane-bound structures, actin- 
and intermediate filaments to the nuclear matrix and chromosomes (Maniotis et al., 1997a, b). Taking this into consideration, it looks but natural that several families of the developmentally important genes can be directly affected by mechanical means, namely by permanent or cyclic tension, pressure or stress relaxation (Resnik et al., 1993; Christen \& Slack, 1999; Kessler et al., 2001; Chiquet et al., 2003; Brouzes \&Farge,2004; Kirwan et al., 2004). Mechanical forces can also affect the transcription rate (D'Addario et al., 2001).

The main aim of this paper was to demonstrate that on the cell and multicelular level mechanical stresses can be coupled with each other in effective feedback permitting production of precise and predictable macromorphological structures from much simpler ones in the absence of any detailed prepattern. Among the possible kinds of feedback, we paid special attention to those generating stress overshoots (HR hypothesis). Further evidence supporting this hypothesis will be presented in accompanying paper (Beloussov et al., 2006).

The HR hypothesis is not absolutely new. It belongs to a fairly large group of physiological and biophysical concepts exploiting the overshoot concept as a consequence of non-linear reactions of the living bodies. To the best of our knowledge the first who proposed it was Arshavsky (1982). In his view, in non-senescent individuals any round of motile activity (including the earliest stages of development) will produce, in the long run, more energy than it has spent: this is just a hyper-restoration loop. The elements of a similar approach can be found in Banes et al. (1995) and Buxbaum and Heidemann (1992) papers.

By emphasizing the mechanical components of morphogenesis, we do not neglect the role of non-mechanical (chemical, freely diffusible) factors. As we have already mentioned, their role may be in specifying the parameters of the morphomechanical feedback: without such a specification no definite morphological structures could be created. However, the role of mechanics in establishing a set of potentially reachable morphogenetical trajectories (chreods, by Waddington (1940) terminology) is, by our suggestion, a leading one. The mechanical components of the morphogenetic machinery deserve, in our view, much more attention than they actually command today.

\section{Acknowledgements}

We thank DrR. Gordon for critically reading the manuscript. This study was supported by the Russian Fund for Fundamental Research, grant \# 05-04-48681.

\section{SUMMARY}

Morphomechanics is a branch of developmental biology, studying the generation, space-time patterns and morphogenetic role of mechanical stresses (MS) which reside in embryonic tissues. All the morphogenetically active embryonic tissues studied in this respect have been shown to bear substantial mechanical stresses of tension or pressure. MS are indispensable for organized cell movements, expression of a number of developmentally important genes and the very viability of cells. Even a temporary relaxation of $M S$ leads to an increase in the morphological variability and asymmetry of embryonic rudiments. Moreover, MS may be among the decisive links of morphogenetic feedback required for driving forth embryonic development and providing its regular space-time patterns. We hypothesize that one such feedback is based upon the tendency of cells and tissues to hyperrestore (restore with an overshoot) their MS values after any deviations, either artificial or produced by neighboring morphogenetically active tissues. This idea is supported by a number of observations and experiments performed on the tissue and individual cell levels. We describe also the models demonstrating that a number of biologically realistic stationary shapes and propagating waves can be generated by varying the parameters of the hyperrestoration feedback loop. Morphomechanics is an important and rapidly developing branch of developmental and cell biology, being complementary to other approaches.

KEY WORDS: morphogenesis, mechanical stress, developmental feedback, morphogenetic model

\section{References}

ALBERTS, B., BRAY, D., LEWIS, J., RAFF, M., ROBERTS, K. and J.D. WATSON (2003). Molecular Biology of the Cell. Garland Publ. Inc. N.Y.

ARNOLDS, W., VAN DEN BIGGELAAR, J. and VERDONK, N. (1983). Spatial aspects of cell interactions involved in the determination of dorsoventral polarity in equally cleaving Gastropoda and regulative abilities of their embryos as studied by micromere deletion in Lymnaea and Patella. W. Roux's Arch. Dev. Biol. 192: 75-85.

ARSHAVSKY, I.A. (1982). Physiological mechanisms and regularities of individual development. Nauka, Moskva, 209 p. (in Russian).

BALABAN, N.Q., SCHWARTZ, U.S., RIVELINE, D., GOICHBERG, P., TZUR, G., SABANAY, I., MAHALU, D., SAFRAN, S., BERSHADSKY, A., ADDADI, L., GEIGER, B. (2001). Force and focal adhesion assembly: a close relationship studied using elastic micropatterned substrates. Nature Cell Biol. 3: 466-472.

BANES, A.J., TSUZAKI, M., YAMAMOTO, J., FISCHER, T., BRIGMAN, B., BROWN, T and MILLER, L. (1995). Mechanoreception at the cellular level: the detection, interpretation and diversity of responses to mechanical signals. Biochem. Cell Biol. 73: 349-365.

BELINTZEV B.N., BELOUSSOV L.V., ZARAISKY A.G. (1987). Model of pattern formation in epithelial morphogenesis. J. Theor.Biol., 129, 369-394.

BELLAIRS, R., BROMHAM, D.R. and WYLIE, C.C. (1967). The influence of the area opaca on the development of the young chick embryo. J. Embryol. Exp. Morphol. 17: 197-212.

BELOUSSOV L.V. (1998) The Dynamic Architecture of a Developing Organism Kluwer Academic Publishers. Dordrecht, Boston, London. $238 \mathrm{P}$.

BELOUSSOV, L.V., LOOUCHINSKAYA, N.N., ERMAKOV, A.S. and GLAGOLEVA, N.S. (2006). Gastrulation in amphibian embryos, regarded as a succession of biomechanical feedback events. Int. J. Dev. Biol. 50: 113-122.

BELOUSSOV, L.V., DORFMAN, J.G., CHERDANTZEV, V.G. (1975) Mechanical stresses and morphological patterns in amphibian embryos. J. Embryol. exp. Morphol. 34: 559-574.

BELOUSSOV, L.V. and V.I. GRABOVSKY (2003). A geometro-mechanical model for pulsatile morphogenesis. ComputerMethods in Biomechanics and Biomedical Engineering. 6: 53-63.

BELOUSSOV, L.V. and V.I. GRABOVSKY (2005). A common biomechanical model for the formation of stationary cell domains and propagating waves in the developing organisms. Computer Methods in Biomechanics and Biomedical Engineering 8: 381-391.

BELOUSSOV L.V., JU.A.LABAS, N.I.KAZAKOVA (1993) Growth pulsations in hydroid polyps: kinematics, biological role and cytophysiology. In: Oscillations and Morphogenesis (L.Rensing ed) Marcel Dekker Basel Hong Kong pp. 183193.

BELOUSSOV L.V., LOUCHINSKAIA N.N., STEIN A.A. (2000) Tension-dependent collective cell movements in the early gastrula ectoderm of Xenopus laevis embryos. Dev. Genes and Evol. 210: 92-104.

BELOUSSOV L.V., SAVELIEV S.V., NAUMIDI I.I., NOVOSELOV V.V. (1994). 
Mechanical stresses in embryonic tissues: patterns, morphogenetic role and involvement in regulatory feedback. Intern. Rev. Cytol. 150: 1-34.

BEREITER-HAHN, J. (1987) Mechanical principles of architecture of eukaryotic cells. In: (J. Bereiter-Hahn, O.R.Anderson and W.-E. Reif eds) Cytomechanics. Springer Verlag, Berlin, Heidelberg, pp. 3-30.

BROUZES, E. and E. FARGE (2004) Interplay of mechanical deformations and patterned gene expression in developing embryos. Curr. Op. Genetics \& Deve/ 14: 367-374.

BUXBAUM, R.E. AND HEIDEMANN, S.R. (1992). An absolute rate theory model for tension control of axonal elongation. J. Theor. Biol. 155: 409-426.

CHERDANTSEVA V.G. (2006) The dynamic geometry of mass cell movements in animal morphogenesis. Int. J. Dev. Biol. 50: 169-182.

CHERDANTSEVA E.M. and CHERDANTSEVA V.G. (2006) Geometry and mechanics of teleost gastrulation and the formation of the primary embryonic axes. Int. J. Dev. Biol. 50: 157-168.

CHIQUET, M., RENEDO, A.S., HUBER, F. and FLUCK, M. (2003) How do fibroblasts translate mechanical signals into changes in extracellular matrix production? Matrix Biology 22: 73-80.

CHOI, S.G., HAN, J.K. (2002) Xenopus Cdc42 regulates convergent extension movements during gastrulation through Wnt/Frizzled signaling pathway. Dev Biol. 244: 342-357.

CHRISTEN B, SLACK JM (1999). Spatial response to fibroblast growth factor signalling in Xenopus embryos. Development 126: 119-125.

CHRZANOWSKA-WODNICKA M., BURRIDGE K. (1996). Rho-stimulated contractility drives the formation of stress fibers and focal adhesions. J Cell Biol. 133: 1403-1415.

COULOMBRE A.J. (1956) The role of intraocular pressure in the development of the chick eye. J. Exp. Zool. 133: 211-226.

COWIN, S.C. (2000). How is a tissue built? J. Biomech. Engineering 122: 553-569.

D'ADDARIO, M., P.D. ARORA, J. FAN, B. GANSS, R.P. ELLEN C.A. and MCCULLOCH (2001). Cytoprotection against mechanical forces delivered through beta 1 integrins requires induction of filamin A. J Biol Chem 276(34): 31969-31977.

DIECKGRAEFE, B,K, WEEMS, D.M, SANTORO, S.A, ALPERS, D.H (1997). ERK and p38 MAP kinase pathways are mediators of intestinal epithelial woundinduced signal transduction. Biochem Biophys Res Commun 233: 389-394.

ERMAKOV, S.A., BELOUSSOV, L.V. (1998) Morphogenetical and differentiation consequences of the relaxation of mechanical tensions in Xenopus laevis blastula. Ontogenez (Russ J. Devel Biol) 29: 450-458.

EZZEL R.M., GOLDMAN W.H., WANG N., INGBER D.E. (1997). Vinculin promotes cell spreadings by mechanical coupling integrins to the cytoskeleton. Exp Cell Res. 231:14-26.

GREEN, P.B., STEELE C.S., RENNICH, S.C. (1996) Phyllotactic patterns: a biophysical mechanism for their origin. Ann. Bot. 77: 515-527.

HARRIS, A.K., STOPAK D., WARNER P. (1984) Generation of spatially periodic patterns by a mechanical instability: a mechanical alternative to the Turing model. J. Embryol. Exp. Morphol. 80: 1-20.

HOUCHMANZADEH, B., E. WEISHAUS, S. LEIBLER (2002). Establishment of developmental precision and proportions in the early Drosophila embryo. Nature 415: 798-802.

HUANG S., INGBER D.E. (2000) Shape-dependent control of cell growth, differentiation and apoptosis: switching between attractors in cell regulatory networks. Expt/ Cell Res. 261: 91-103

HUNTER, T. (2000). Signaling - 2000 and beyond. Cell. 100: 113-127.

IVANENKOV, V.V., MININ, A.A., MESCHERYAKOV, V.N. and MARTYNOVA, L.E. (1990). Surface polarization in loach eggs and two-cell embryos: correlation between surface relief, endocytosis and cortex contractility. Int. J. Dev Biol. 34: 337-349.

JACOBSON, A.G. and GORDON, R. (1976). Changes in the shape of the developing vertebrate nervous system analyzed experimentally, mathematically and by computer simulation. J.Exp.Zool. 197: 191-246.

JAEGER, J., SURKOVA, S., BLAGOV, M., JANSSENS, H., KOSMAN, D., KOZLOV, K., MANU, MYASNIKOVA E., VANARIO-ALONSO, C.E., SAMSONOVA, M., SHARP, D.H., REINITZ, J. (2004). Dynamic control of positional information in the early Drosophila embryo. Nature 430: 368-371.

KAUFFMAN, S. A. (1993) The Origins of Order. O.U.P., N.Y.

KAVERINA, O. KRYLYSHKINA, K. BENINGO, K. ANDERSON, YU-LI WANG and J.V. SMALL (2002) Tensile stress stimulates microtubule outgrowth in living cells. J. Cell Science 115: 2283-2291.

D. KESSLER, S. DETHLEFSTEN, I. HAASE, M. PLOMANN, F. HIRCHE, T. KRIEG and B. ECKES (2001) Fibroblasts in mechanically stressed collagen lattices assume a "synthetic" phenotype. J. Biol. Chemistry 276 (39): 36575-36585.

KIEHART D.P., C.G. GALBRAITH, K.A. EDWARDS, W.L. RICKOLL, R.A. MONTAGUE (2000) Multiple forces contribute to cell sheet morphogenesis for dorsal closure in Drosophila. J. Cell Biol. 149: 471-490.

KIRWAN, R.P., CREAN, J.K., FENETRY, C.H., CLARK, A.F., O'BRIEN, C.J. (2004) Effect of cyclical mechanical stretch and exogeneous transforming growth factor $\beta$ - 1 on matrix metalloproteinase- 2 activity in lamina cribrosa cells from the human optic nerve head. J. Glaucoma 13 (4): 327-334.

KOEHL, M.A.R. (1990). Biomechanical approaches to morphogenesis. Sem. Devel. Biol. 1, 367-378.

KOLEGA, J. (1986) Effects of mechanical tension on protrusive activity and microfilament and intermediate filament organization in an epidermal epithelium moving in culture. J. Cell Biol. 102: 1400-1411.

KRAIN B., NORDHEIM A. (1999). Artefactual gene induction during preparation of Xenopus laevis animal cap explants. Int J Dev Bio/43: 563-566.

KRAUS, Y.A. (2006). Morphomechanical programming of morphogenesis in Cnidarian embryos. Int. J. Dev. Biol. 50: 267-275.

KUCERA, P., MONNET-TSCHUDI, F. (1987). Early functional differentiation in the chick embryonic disc: interactions between mechanical activity and extracellular matrix. J. Cell Sci Supp/8: 415-432.

LANDAU L. D., LIVSHITZ E. M. (1976), Theory of Elasticity. Nauka, åoscow (In Russian).

MANIOTIS A.J., CHEN C.S., INGBER D.E. (1997). Demonstration of mechanical connections between integrins, cytoskeletal filaments and nucleoplasm that stabilize nuclear structure. Proc Nat/ Acad Sci USA 94: 849-854.

MARTYNOV, L.A. (1982) The role of macroscopic processes in morphogenesis. In: Mathematical Biology of Development (A.I.Zotin, E.V.Presnov eds) Nauka, Moskva pp. 135-154 (in Russian).

ODELL, G.M., OSTER, G., ALBERCH, P., BURNSIDE, B. (1981) The mechanical basis of morphogenesis. I. Epithelial folding and invagination. Devel. Biol. 85: 446-462.

PLETJUSHKINA O.J, BELKIN A.M, IVANOVA O.J, OLIVER T., VASILIEV J.M, JACOBSON K. (1998). Maturation of cell-substratum focal adhesions induced by depolymerization of microtubules is mediated by increased cortical tension. // Cell Adhesion and Communication. 5: 121-135.

PRIGOGINE, I. (1980) From Being to Becoming. W.H. Freeman and Company. N.Y.

RESNICKN., COLLINS, T., ATKINSON, W., BONTHRON, D.T., DEWEY, C.F. and GIMBRONE, M.A. (1993). Platelet-derived growth factor B chain promoter contains a cis-acting fluid shear-stress-responsive element. Proc. Nat. Acad. Sci. USA 90: 4591-4595.

RIVELINE, D., ZAMIR, E., BALABAN, N. Q., SCHWARZ, U. S., ISHIZAKI, T., NARUMIYA S., KAM, Z., GEIGER, B. AND BERSHADSKY, A. D. (2001). Focal Contacts as Mechanosensors: Externally Applied Local Mechanical Force Induces Growth of Focal Contacts by an mDia1-dependent and ROCK-independent Mechanism. J. Cel/ Biol. 153: 1175-1185.

SKIBBENS, R.V. and E.D.SALMON (1994). Kinetochore directional instability in vertebrate mitotic cells. In: Biomechanics of Active Movement and Division of Cells (N.Akkas ed.) Springer-Verlag Berlin etc PP. 545-550.

STERN, C.D. (1984) A simple model for early morphogenesis. J. Theor. Biol. 107: 229-242.

THOM, R. (1972). Structuralism in biology. In: Towards the Theoretical Biology (C.H. Waddington ed.) Edinburgh University Press, Edinburgh.

THOMPSON, D'ARCY (2000). On Growth and Form. Cambridge University Press, Cambridge.

TSIKOLIA, N. (2006). The role and limits of gradient based explanation of morphogenesis: a theoretical consideration. Int. J. Dev. Biol. 50: 333-340. 
WADDINGTON, C.H. (1940). Organizers and Genes. Cambridge Univ. Press, Cambridge.

WALLINGFORD J.B., S.E. FRASER and R.M.HARLAND (2002). Convergent extension: the molecular control of polarized cell movement during embryonic development. Developmental Cel/2: 695-706.

WANEKN., MARCUM B.A., LEE H.T., CAMPBELL R.D. (1980) Effect of hydrostatic pressure on morphogenesis in nerve-free hydra. J. Exp. Zool. 211: 275-280.

WATTERSON J.G. (2004). Enzyme function: random events or coherent action? http://www.1sbu.ac.uk/water/

WILSON P.A., OSTER G and KELLER R. (1989) Cell rearrangements and segmentation in Xenopus: direct observations of cultured explants. Development 105 : 155-166. 